\title{
Method of the drift: More than just an optimization method
}

\author{
Olivier Demars ${ }^{1}$ and Christophe Dubois ${ }^{1}$ \\ ${ }^{1}$ Deltamu, Metrology Consulting \& training, 63800 Cournon d'Auvergne, France
}

\begin{abstract}
The French guidelines FD X07-014[1] give different methods of calibration interval which allow to justify intervals of metrological confirmation for the measuring instruments used in a company. It offers three concrete methods. One of them is "Method of the drift". It is a method based on the equipment drift. The first stage of this method consists in modelling the drift observed for every equipment by the method of least squares. Then, all the instruments of a same family are grouped, in order to determine the maximal drift of the family (statistically). Then, the periodicity of every instrument can be calculated according to its last value of calibration and the maximal drift of its family. This method is used for instruments having a known drift, and essentially for the gauges. So the first aim of this method is to determine the calibration interval. But the purpose of this article is to show that a more detailed analysis can give lots of information and increase the value of the calibration operation. We propose some examples of applications actually implemented in the industry and other that could be easily implemented.
\end{abstract}

\section{Presentation of the method of the drift}

The AFNOR (Association Française de Normalisation French national organization for standardization) proposes a guide, published in 2006, about the optimization and the justification of metrological confirmation for the measuring instruments: FD X017-014. This guidelines proposes three concrete methods to calculate the optimum calibration periodicity. Among these three methods, one of them, the "Method of the drift ", offers to estimate the periodicity of the measuring instruments by using the drift of the measuring instruments. This method is adapted to every instrument having a known drift and mainly for the gauges.

Since the «real» drift of a measuring instrument is impossible to determine (use of the instrument between two calibrations, measurement uncertainties of the calibration laboratories, inhomogeneity of measuring equipments,...), the guide offers to determine the maximum wear (the drift) of an equipment family (same sort). This maximum wear will then be used to determine the individual periodicity of each instrument of the family regarding the last calibration result of one equipment.

\section{Setting up of the method}

The implementation of the drift method is carried out in several stages.

\subsection{Individual wear factor}

The first stage consists in determining the individual wear for each measuring instrument and each measuring group (for example side Go in and side Not Go In for a double instrument), typically by the method of least squares. The wear factor, given by the right slope, represents the observed drift of the measuring instrument over time.

Figure 1: Modeling the drift of a gauge

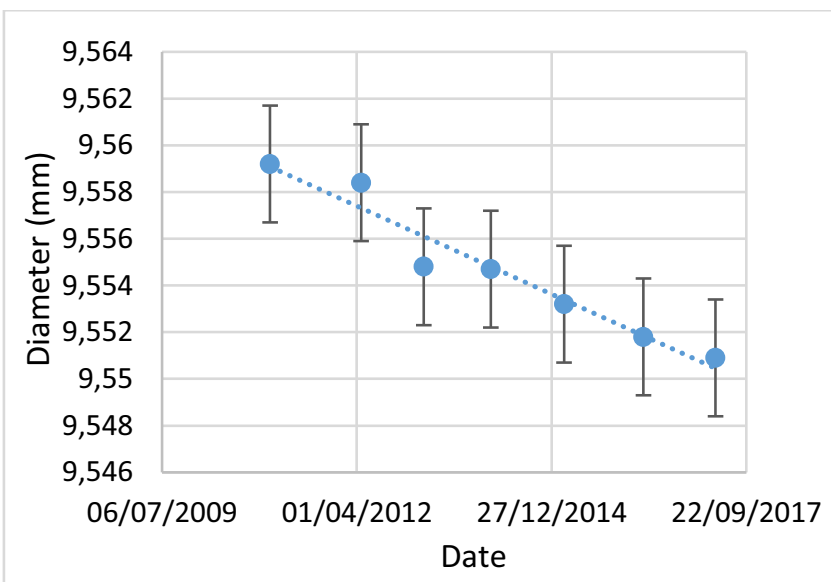

To determine the individual wear factor, it is recommended to have a historical low of three calibration by instrument over a period between the first and the last calibration above a year in order to limit the impact of the calibration uncertainty of the laboratory (a larger number of calibration or a higher duration will allow to refine this cause of wear).

\footnotetext{
* Corresponding author: metrologie@,deltamu.com
}

C The Authors, published by EDP Sciences. This is an open access article distributed under the terms of the Creative Commons Attribution License 4.0 (http://creativecommons.org/licenses/by/4.0/). 


\section{$2.2 \quad$ Family grouping}

Once the drift of each instrument has been determinated, the equipments are grouping by family (designation, type, group ,...) in order to determine the maximum wear of the aforesaid family.

Figure 2: Example of distribution of the drifts of a family

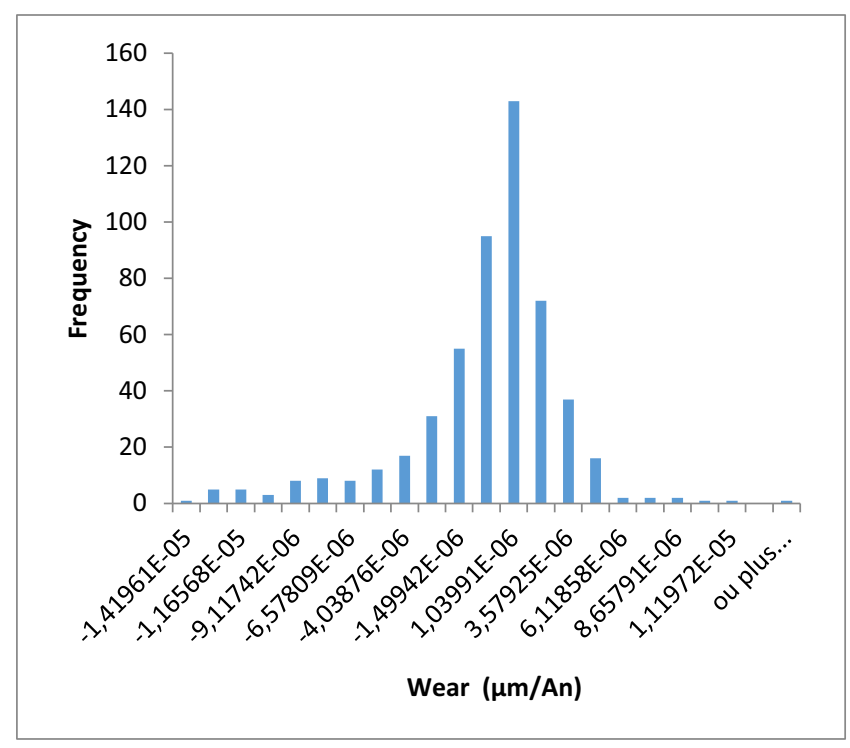

\subsection{Maximal wear factor of a family}

Under the assumption of a normal probability distribution of the drifts, the maximum drift is then calculated by the following formula:

$$
F U \max =F U m o y \pm 2 s
$$

Avec :

$$
\begin{array}{ll}
\text { - } & \text { FUmax : Maximum drift of the family } \\
\text { - } & \text { FUmoy : Medium drift of the family } \\
\text { - } & \pm: \text { Standard deviation of the drift of the family } \\
& \text { positive (example of the rings, snap gauges) the }
\end{array}
$$$$
+ \text { sign will be used }
$$

The factor 2 allows to define a maximum value to the population at $97.5 \%$. Only the $2.5 \%$ of the drifts can be superior to this FUmax.

\subsection{Individual optimal periodicity}

The optimal periodicity of each instrument can then be determined from this maximal drift.
Figure 3 : Use of the maximal drift of a family to determine the calibration periodicity

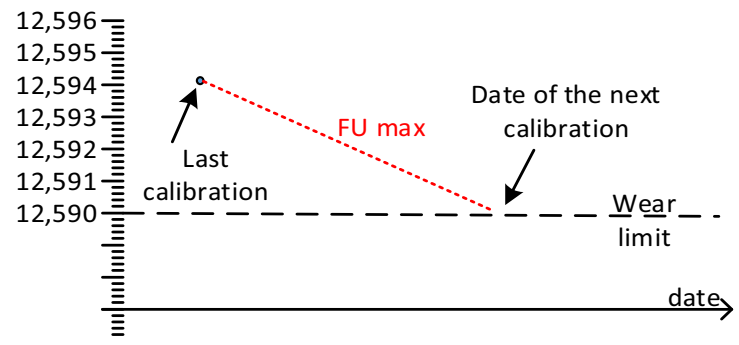

The optimal periodicity is calculated according to the following formula:

$$
\text { PERopti }=(\text { Limit }- \text { Vmes }) / \text { FUmax }
$$

With:

- PERopti : Optimal periodicity of the instrument

- Limit : Limit of use of the instrument

- Vmes : Last value measured for the instrument Using this maximal drift ensures that the instrument will still be in the limits of conformity during the next calibration (on the condition of use in compliance with the recommendations of use)

It is however preferable not to wait for the term calculated, since statistically, $2.5 \%$ of gauges can wear out faster. It is then advised to use an arbitrary safety factor based on the gravity of the consequences of an erroneous measurement. This safety factor can also be a percentage of the periodicity previously calculated:

$$
P E R=\mathrm{k} \cdot P E R o p t i
$$

With :

- PER: Final periodicity of the instrument

- K: Safety factor. This safety factor can for example be equal to $50 \%$ for the side Go In and $80 \%$ for the side Not Go In.

The objective of this method is to propose to companies a solution allowing to determine the optimal periodicities of instruments and thus most of the time to calibrate less often while insuring the conformity of the instrument. The collected information and the work made to set up the method can be easily used to go farther and identify problems before they have an impact on the production.

\section{Smart Metrology: use the available information}

The analysis presented above allows naturally to choose a wise periodicity but it is possible to collect a lot of others interesting information.

Various strategies of analysis are proposed in this paragraph.

\subsection{Atypical family}

In the step of grouping by family (cf. §2.2), it is possible that the histogram of synthesis reveals one subfamily, a group of equipment which has a wear factor different from others (generally stronger). 
Figure 4 : presence of a subfamily

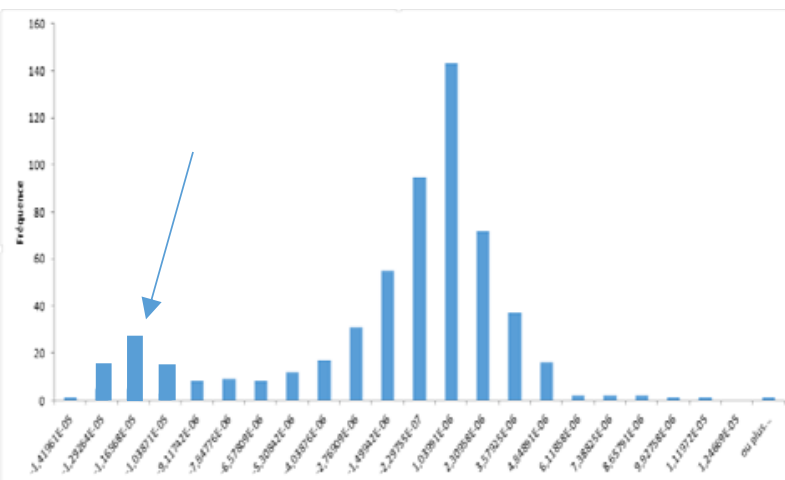

It is then important to understand the origin of this difference: error of grouping, different behavior in a particular service, a type of poor quality gauge, ...

\subsection{Periodicity too short}

To understand, let's take the case of a standard measuring rod, unused. By calibrating it in 1 month apart with an uncertainty of calibration of $1 \mu \mathrm{m}$ (standard uncertainty of a COFRAC calibration laboratory), it is easy to obtain with the calibration results a wear grade of $7 \mu \mathrm{m} /$ year which could let think of a very fast wear of the rod while this one did not evolve since it was not used.

Figure 5 : Case of a calibration periodicity too short (1 month)

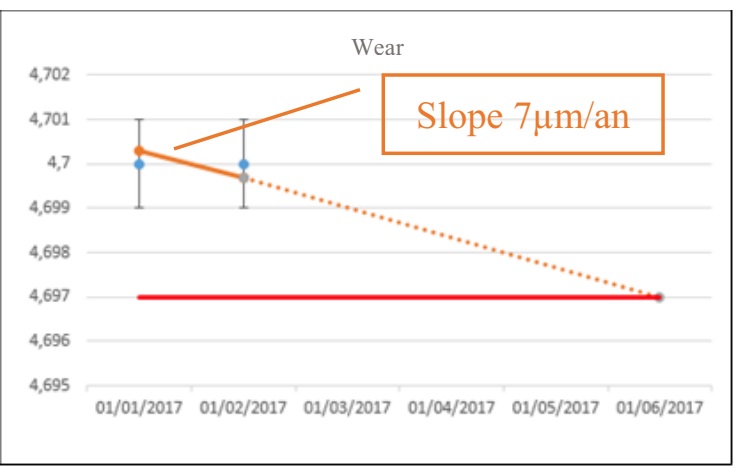

The implementation of a study of the drift, on several gauges and several calibration results, will allow to notice that this calculated "wear" is owed in fact to the uncertainty of the laboratory which, when calibrations are too regularly realized, will have an important impact on this calculated « wear ».
Figure 6 : periodicity longer (1 year), with the same calibration results

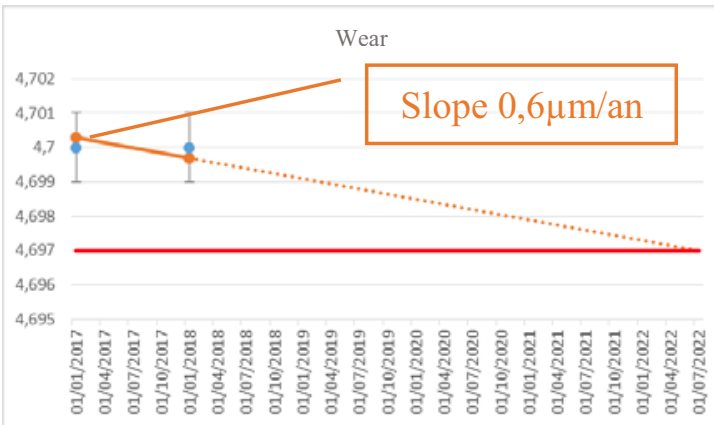

Calibrating the gauges too often can tend to alarm metrologists while there is no reason. Thus a too short periodicity is not inevitably well adapted for this kind of instruments.

\subsection{Monitoring of calibration results}

\subsubsection{Unitarian result "suspect"}

Calibrations are realized by specialists of the measure, so it should not be necessary to verify the values registered on the certificate of calibration.

Nevertheless, the error is human. The measures being realized and often noted by individuals, it is completely possible, for an accredited laboratory or not, to make an error during the measurement or even to measure differently from usually. So, by following the drift of equipments, it is possible to verify the relevance of the results and so prevent any possible atypical deviation of calibration results.

By taking the example of a plain plug gauge, the value of the moderate diameter is supposed normally always to decrease. If the diameter measured (to see figure below) goes back up, it is then possible that there is an error of measurement.

Figure 7 : Case of the evolution of the diameter of a plain plug gauge with a potential measurement error from the laboratory

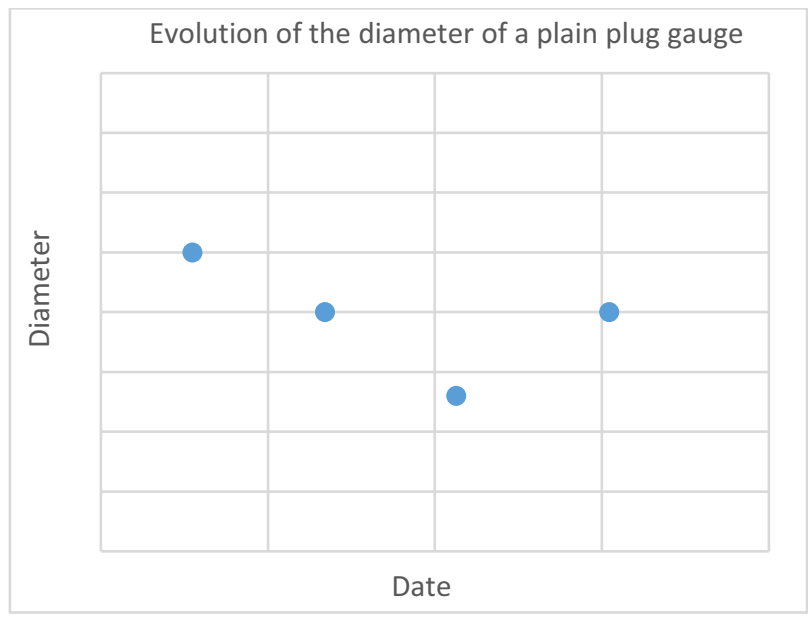

Before questioning the calibration, it is however advisable to take into account its uncertainty of measure. Indeed if the uncertainties measurement recover widely 
on the moderate values, the calibration result cannot be considered atypical.

In the case Figure 8, the uncertainties measurements recover, so there is nothing abnormal to obtain such an evolution of the drift.

Figure 8 : Case of a plain plug gauge with a normal evolution of diameter considering the laboratory uncertainties

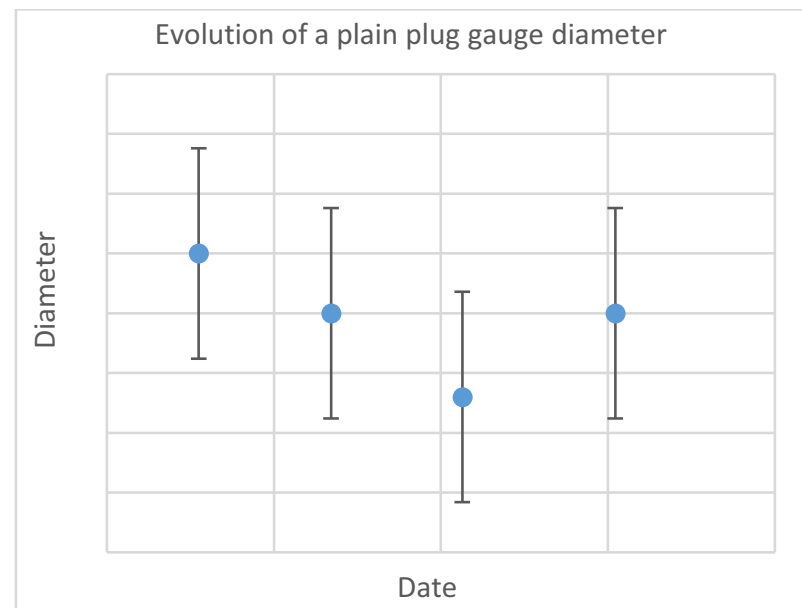

On the contrary in the case Figure 9, it is necessary to lead an investigation and doubtless to ask the laboratory to redo the measure of the equipment.

Figure 9 : Case of a plain plug gauge which diameter evolution can be suspect because of the laboratory uncertainties

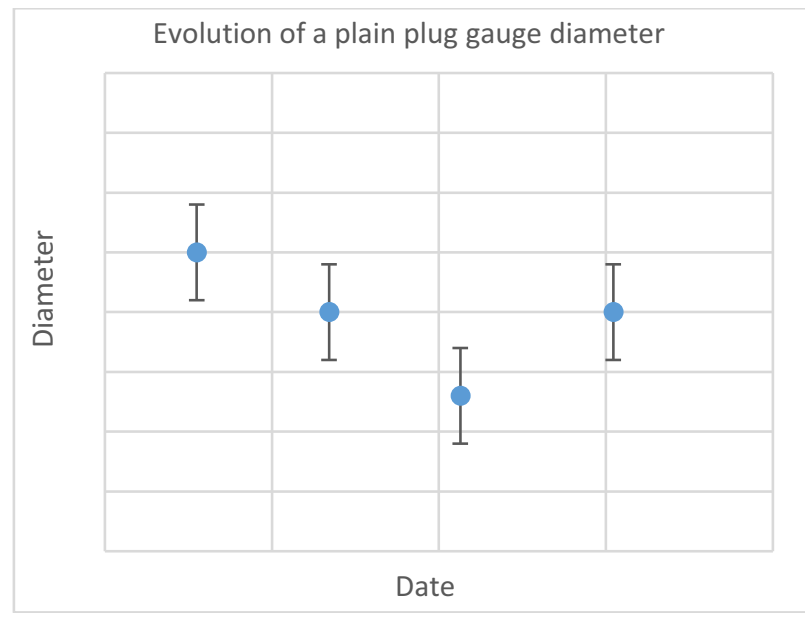

\subsubsection{Several "suspect" results}

It is not rare while sending the instruments in calibration to see, thanks to the implementation of the method of the drift, a whole instrument batches with the same name with the measure results showing the same trend (Increase / decrease of the diameter since the last calibration of the same value, evolution of the instrument in the opposite sense to that waited). If the production did not change since the last calibration, there is a strong probability that these evolutions by family result from modifications which would have been realized within the laboratory of calibration.

The reasons proposed below can be justifications of these evolutions

- Modification of the value of the standard of the laboratory

- Bad regulation of the bench of measure

- Change of the operator

- Change of calibration laboratory

Often, these suspect results have only few impacts on the production but it is however advisable to see with the laboratory if it has a reason to justify them.

\subsection{Wear too fast}

Once the FUmax identified for the implementation of the calibration periodicity, we might as well use them. During a calibration result of a gauge, it can be interesting to compare the wear of the last calibration with the FUmax. If the wear is superior to the FUmax then there might be a problem:

- The calibration laboratory made a mistake

- There is a problem with the gauge in the company (bad use, bad quality gauge...) mauvaise qualité, ...)

\section{Figure 10 : Wear too fast}

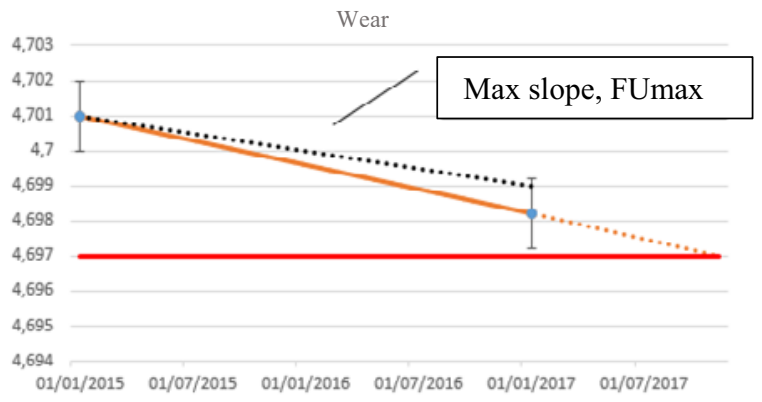

This analysis allows to identify a potential problem on a gauge before its unconformity in order to avoid a bad impact on the production.

Remark: in the application of this method at our customers, for the moment all the noticed atypical grades had for origin an error of calibration.

\subsection{Work with number of use}

The method was introduced with a use of the wear in the time of the gauges. It is completely possible to use the same principle but to argue in wear according to the number of use. This approach is possible when the gauge is associated with a production measurement very precise. From the number of produced pieces, we can deduct a number of use of the gauge. The periodicity of calibration is expressed then not in date, but in number of remaining use.

This information can be then used in the choice of an equipment when an order of manufacturing is made. If I have to produce 5000 pieces and the gauge has a remaining use of 8000 then I can use it. However if the 
same order of manufacturing is made again I will not be able to use this gauge without any risk.

This approach in at least 2 advantages. It allows to avoid calibrating the unused gauges. And it allows to choose a way adapted to the production volume, and to avoid finding itself with a not conform mean at the end of production.

On the other hand it requires a management of the ways associated with the order of manufacturing.

\subsection{Capitalization and improvement}

\subsubsection{Refin the FUmax}

During the implementation of the method, the first FUmax calculated is generally not optimum. Indeed, the study is generally made over a short period (2 years) because the history is either difficult to access (with difficulty exploitable paper format), or doubtful (the company judges that things were able to evolve and do not wish to use information too old). In this case, the uncertainty of the laboratory is strongly present in the FUmax (see §3.2). The FUmax is increased. Over time and a wise capitalization of calibration data, it becomes possible to re-estimate the FUmax with more data and at a longer time.

The impact of the uncertainty of calibration of the laboratory is reduced, so as the FUmax.

Example: by simulation Monte Carlo [2], it is possible to estimate the impact of the calibration uncertainty on the estimation of FUmax.

1- Suppose a family of rods not used and calibrated in 1 year of interval by a laboratory with an uncertainty of $1 \mu \mathrm{m}$. Because of the uncertainties, the successive calibrations do not give an identical result, a wear factor (artificial because rods are not used) can be estimated. The FUmax outcome of such a study, by simulation Monte Carlo, is of $-1,4 \mu \mathrm{m} /$ year (owed only to the calibration uncertainties).

2- Let us suppose now that we have 2 additional calibrations (total history over 3 years) and that we decide to redo the study. The FUmax, still by simulation Monte Carlo, then passed to $-0,4$ $\mu \mathrm{m} /$ year. The calibration uncertainties have less impact.

This study gives a rough estimate of the impact of the calibration uncertainty. In the reality, on the real family of used rods, it is normal to see the FUmax decreasing when the FUmax is re-estimated after a few years and with a history more consequent of calibration.

\subsubsection{Inter compagnies comparisons}

Sharing of FUmax of several companies can be an interesting source of improvement.

Each company can compare its FUmax with the other ones[3] and identify a possible abnormal behavior (unjustified important wear for example) and set axes of improvement (training, investment in gauges carbides). A company having a not exploitable history (few calibres, $\mathrm{CV}$ of calibration in paper) could use an upper bound FUmax of a similar company and, later, recalculate its own FUmax.

These are two commonly used uses by Deltamu during the park management of its customers.

\section{Smart Metrology: a tool for the production}

The implementation of an optimization by the method of the drift can also allow to identify abnormal behavior in production. An instrument which would have an individual wear very superior to the maximal drift of the family it belongs to could alert the metrologist on a concern during the measure with this equipment. Various problems can be highlighted:

- $100 \%$ controls of a production with a means. In this case the wear is necessarily very important and a periodic calibration seems then useless. It is necessary to anticipate these particularly aggressive uses for the equipment to avoid non-compliance inconvenient. - A change of behavior of wear of the calibre can highlight a change of the production: formation defect of new operators, change of the nature of the piece produced (decrease of diameter, more abrasive material...)

In its history, it happened to Deltamu to meet that kind of production concern. For example, during a study of optimization for a customer, Deltamu highlighted a group of instruments which tended to derive more quickly than the other instruments of the same family. The customer produced tappings with drills, which he controlled then to $100 \%$ with thread plug gauges. Drills wearing out slowly, they produced smaller and smaller holes.

Gradually, the diameter of the hole gets closer to the diameter of the thread which is enormously used when diameters are "synchronized" without the operator notices it, the phenomenon being very progressive. At the end, the thread accepted too narrow holes. The implementation of an expertise and a study of the drift allowed to set up corrective actions needed to correct this detected problem.

\section{Conclusion}

Metrology is not only the management of the park or the calibration of measuring instruments. This is only a minor part of its work. This article shows that by exploiting the data and the provided tools, the metrologist can supply a lot of useful information [4] for the whole company.

\section{References}

1. Afnor, FD X07-014, Métrologie - Optimisation des intervalles de confirmation métrologique des équipements de mesure (2006)

2. ISO/CEI GUIDE 98-3/S1, Uncertainty of measurement -- Part 3: Guide to the expression of uncertainty in measurement (GUM:1995) 
Propagation of distributions using a Monte Carlo method (2008)

3. ISO 5725, Accuracy (trueness and precision) of measurement methods and results (1994)

4. J.M. Pou, http://www.smart-metrology.com, Les étalonnages coûtent chers, autant qu'ils servent ! (2014) 